\title{
D-cycloserin, a NMDA-agonist may be a treatment option for anti-NMDAR encephalitis
}

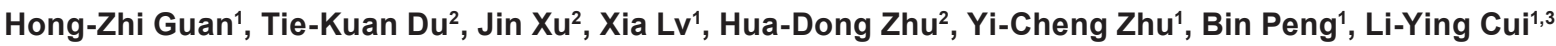 \\ ${ }^{1}$ Department of Neurology, Chinese Academy of Medical Sciences and Peking Union Medical College, Beijing 100730, China. \\ ${ }^{2}$ Department of Emergency, Chinese Academy of Medical Sciences and Peking Union Medical College, Beijing 100730, China. \\ ${ }^{3}$ Neuroscience Center, Chinese Academy of Medical Sciences and Peking Union Medical College, Beijing100730, China.
}

Correspondence to: Dr. Bin Peng, Department of Neurology, Chinese Academy of Medical Sciences and Peking Union Medical College, Beijing 100730, China. E-mail: pumchpengb@163.com

How to cite this article: Guan HZ, Du TK, Xu J, Lv X, Zhu HD, Zhu YC, Peng B, Cui LY. D-cycloserin, a NMDA-agonist may be a treatment option for anti-NMDAR encephalitis. Neuroimmunol Neuroinflammation 2016;3:189-91.

\begin{tabular}{l} 
Article history: \\
Received: $15-06-2016$ \\
Accepted: $12-07-2016$ \\
Published: $31-08-2016$ \\
\hline Key words: \\
D-cycloserine \\
encephalitis \\
autoantibody \\
N-methyl-D-aspartate receptor \\
agonist
\end{tabular}

\section{INTRODUCTION}

Anti-N-methyl-D-aspartate receptor (NMDAR) encephalitis is caused by reversible neuron dysfunction associated autoantibodies against NMDAR. ${ }^{[1,2]}$ Most cases with anti-NMDAR encephalitis respond to immunotherapy. However, some cases are refractory to immunotherapy and have prolonged intensive care unit duration. ${ }^{[1]}$ Specific symptomatic treatment for anti-NMDAR

\begin{abstract}
Anti-N-methyl-D-aspartate receptor (NMDAR) encephalitis is caused by reversible neuron dysfunction associated an autoantibody-mediated decrease of NMDAR in the entire brain. A N-methyl-D-aspartate (NMDA) -agonist treatment for anti-NMDAR encephalitis might have a role considering its specific mechanism. The authors used D-cycloserine, a partial NMDA-agonist in a refractory case with prolonged intensive care unit duration. A 13-year-old female presented with headache, cognitive deterioration, generalized seizures, coma and hypoventilation with required mechanical ventilation. Anti-NMDAR antibodies were identified in cerebrospinal fluid and serum confirming anti-NMDAR encephalitis. The patient was refractory to firstline and second-line immunotherapy and removal of ovary teratoma. D-cycloserine was then administered and her symptoms improved gradually and significantly. This is the first reported case in which D-cycloserine was applied to this disease. D-cycloserine might be a potential option as specific treatment in anti-NMDAR encephalitis.
\end{abstract}

encephalitis might have a role considering the mechanism of NMDAR dysfunction in the disorder. However, the current symptomatic medication, such as anti-epileptics and neuroleptic are usually less effective in anti-NMDAR encephalitis. Therefore, we think a $\mathrm{N}$-methyl-D-aspartate (NMDA)-agonist might be used to reverse its neuron dysfunction. We reviewed the literatures on clinical application of NMDA-agonists and found that D-cycloserine, a 
partial NMDA-agonist, may be a reasonable option. We then applied the medicine to a patient with antiNMDAR encephalitis refractory to immunotherapy. The patient's parents signed informed consent for publication of this report.

\section{CASE REPORT}

A previously healthy 13-year-old female presented with headache and nausea without fever. One week later, she progressively developed agitation, cognitive deterioration, generalized seizures, coma and hypoventilation with required mechanical ventilation. Brain magnetic resonance imaging was normal. Cerebrospinal fluid (CSF) analysis demonstrated pleiocytosis ( 180 white cells/ $\mu \mathrm{L}$ ) with a normal protein and glucose concentration. Anti-NMDAR antibodies was identified in CSF and serum confirming antiNMDAR encephalitis. The patient was initially treated with intravenous immumoglobulin (IVIg) and methylprednisolone with no improvement. Four weeks after the onset, ovary teratoma was identified on ultrasound and laparoscopic resection was performed with pathological diagnosis of teratoma. After the operation another 2 cycles of IVIg, cyclophosphamide (IV $500 \mathrm{mg} / \mathrm{m}^{2}$ monthly) and mycophenolate mofetil $(1,000 \mathrm{mg} / \mathrm{d})$ was added because of insufficient clinical response. Five months after initial presentation, no remarkable clinical improvement was observed. The patients were still unconscious with recurrent seizure, chorea, tackycardia and episodes of hypoventilation requiring mechanical ventilation. D-cycloserine was then administered $125 \mathrm{mg} / \mathrm{d}$ for 1 week followed $250 \mathrm{mg} / \mathrm{d}$. Two weeks after the first administration of D-cycloserine, her symptoms improved gradually. Seizure and choreic movements were reduced and hypoventilation disappeared, leading to discharge from the intensive care unit. Two weeks later, a further improvement was observed. She was able to understand simple orders. Seizure and chorea had disappeared. D-cycloserine was reduced to $125 \mathrm{mg} / \mathrm{d}$ and then stopped. The patient is functionally normal now with modified Rankin Score 0 at her last follow-up 1 year from disease onset.

\section{DISCUSSION}

We report a case of severe anti-NMDAR encephalitis refractory to immunotherapy and response to $\mathrm{D}$-cycloserine. This is the first reported case in which this NMDA-agonist was applied to this disease. Our observation indicates that D-cycloserine may be a therapeutic option for anti-NMDAR encephalitis.

The cellular mechanisms of anti-NMDAR encephalitis are a specific and reversible decrease of NMDAR surface density due to immunoglobulin G-mediated internalization. The internalization is caused by crosslinking of the receptors by the autoantibodies. ${ }^{[2]}$ As we know, antibody-mediated receptor internalization had been demonstrated for myasthenia gravis. ${ }^{[3]}$ Symptomatic treatment with pyridostigmine in myasthenia gravis is relatively specific considering its mechanism. It is reasonable for neurologist to find and apply a NMDAagonist to treatment of anti-NMDAR encephalitis.

D-cycloserine, known as anti-tuberculotic medicine, has been widely introduced to neuropsychiatric studies, since its central activation mechanism as a partial NMDA-agonist has been found. ${ }^{[4,5]}$ Increasing evidence suggests that D-cycloserine may be effective in various psychiatric diseases, including schizophrenia, anxiety disorders, addiction, major depression and autism as well as in neurological diseases, including dementia, Alzheimer's disease and spinocerebellar degeneration. ${ }^{[5,6]} \mathrm{D}$-cycloserine acts at the glycine-binding site of the NMDA receptor, which is located at its NR1 subunit. As a partial agonist, D-cycloserine acts like an agonist at low doses but has antagonistic features with high doses. The doses of D-cycloserine used for modulation of neuroplasticity are lower than for antituberculotic indication. The typical application in antituberculosis therapy is 250 to $500 \mathrm{mg}$ twice daily. In the neuropsychiatric studies, D-cycloserine was administrated at a dose of $50 \mathrm{mg} / \mathrm{d}$ to $250 \mathrm{mg} / \mathrm{d}^{[5,6]}$ The pharmacological properties of D-cycloserine indicate its potential as central nervous system modulator. The maximum concentration in blood is reached $2 \mathrm{~h}$ after oral application. About $54-79 \%$ of oral intake reaches the CSF. ${ }^{[7,8]}$

There are some interesting observations in which NMDA antagonist including ketamine may improve symptoms of anti-NMDAR encephalitis. ${ }^{[9]}$ However, the administration of NMDA antagonist to anti-NMDAR encephalitis is not consistent with the mechanism of NMDAR hypofunction in the disorder and in our practice there were not obvious improvement after administration of ketamine or memantine to our patients with anti-NMDAR encephalitis. Recently, Heresco-levy et al. ${ }^{[10]}$ reported the clinical and electrophysiological effects of D-serine in a schizophrenia patient positive for anti-NMDAR antibodies. D-serine was administrated in 6 weeks to their patient in which dose were increased gradually from 1.5 to $4 \mathrm{~g} /$ day.

This preliminary study is limited by the fact that spontaneous remission of symptoms even after severe and long disease duration is still expected in 
anti-NMDAR encephalitis while the treatments with cyclophosphamide and mycophenolate may take 3-4 months to have responses. We cannot draw conclusions that the clinical improvement was a consequence of the D-cycloserine treatment.

In conclusion, D-cycloserine seems to be a promising symptomatic treatment for anti-NMDAR encephalitis. Further studies are warranted to evaluate the effectiveness of D-cycloserine. Understanding the mechanisms underlying the disorder will lead to discover novel therapy including other NMDA-agonists.

\section{Acknowledgments}

We thank our patient and her family.

\section{Financial support and sponsorship} Nil.

\section{Conflicts of interest}

There are no conflicts of interest.

\section{Patient consent \\ Obtained.}

\section{Ethics approval}

The patient was treated within the standards of our institute and the report was approved.

\section{REFERENCES}

1. Dalmau J, Lancaster E, Martinez-Hernandez E, Rosenfeld MR, Balice-
Gordon R. Clinical experience and laboratory investigations in patients with anti-NMDAR encephalitis. Lancet Neurol 2011;10:63-74.

2. Moscato EH, Peng X, Jain A, Parsons TD, Dalmau J, Balice-Gordon RJ. Acute mechanisms underlying antibody effects in anti-N-methyl-Daspartate receptor encephalitis. Ann Neurol 2014;76:108-19.

3. Sideris S, Lagoumintzis G, Kordas G, Kostelidou K, Sotiriadis A, Poulas K, Tzartos SJ. Isolation and functional characterization of antiacetylcholine receptor subunit-specific autoantibodies from myasthenic patients: receptor loss in cell culture. J Neuroimmunol 2007;189:111-7.

4. Watson GB, Bolanowski MA, Baganoff MP, Deppeler CL, Lanthorn TH. D-cycloserine acts as a partial agonist at the glycine modulatory site of the NMDA receptor expressed in Xenopus oocytes. Brain Res 1990;510:158-60.

5. Schade S, Paulus W. D-Cycloserine in Neuropsychiatric Diseases: A Systematic Review. Int J Neuropsychopharmacol 2016; doi: 10.1093/ ijnp/pyv102.

6. Storch EA, Murphy TK, Goodman WK, Geffken GR, Lewin AB, Henin A, Micco JA, Sprich S, Wilhelm S, Bengtson M, Geller DA. A preliminary study of D-cycloserine augmentation of cognitivebehavioral therapy in pediatric obsessive-compulsive disorder. Biol Psychiatry 2010;68:1073-6.

7. Zhu M, Nix DE, Adam RD, Childs JM, Peloquin CA. Pharmacokinetics of cycloserine under fasting conditions and with high-fat meal, orange juice, and antacids. Pharmacotherapy 2001;21:891-7.

8. D'Souza DC, Gil R, Cassello K, Morrissey K, Abi-Saab D, White J, Sturwold R, Bennett A, Karper LP, Zuzarte E, Charney DS, Krystal JH. IV glycine and oral D-cycloserine effects on plasma and CSF amino acids in healthy humans. Biol Psychiatry 2000;47:450-62.

9. MacMahon M, Naysmith MR, McCallion S, Rhodes J. Ketamine infusion associated with improved neurology in a patient with NMDA receptor encephalitis. Case Rep Crit Care 2013;2013:383125.

10. Heresco-Levy U, Durrant AR, Ermilov M, Javitt DC, Miya K, Mori H. Clinical and electrophysiological effects of D-serine in a schizophrenia patient positive for anti-N-methyl-D-aspartate receptor antibodies. Biol Psychiatry 2015;77:e27-9. 